\title{
Study on fracture fixation in comorbid conditions
}

\author{
Dr. M.Prasanth ${ }^{1}$,Dr. P. Anil Babu \\ ${ }^{1,2}$ (DepartmentOf Orthopaedics, GMC /GGH, Guntur ,AP,India)
}

\begin{abstract}
There are many co morbid conditions which exist before a fracture is sustained and developed or identified during the evaluation for fitness to undergo surgery. The study is done with 83 patients who are diagnosed as having a co morbid condition which can influence the outcome preoperatively and post operatively. They are grouped in to three groups where in cardiac, respiratory and renal comorbid conditions are identified. Each co morbid condition is evaluated and treated by the concerned medical speciality for the treatment as well as to ascertain the fitness to undergo surgery. The fracture patterns in this study are cases which require definitive fixation as and when the patients are fit to undergo surgery. The ideal time of fracture fixation with ideal implant is around five days, beyond which the fracture reduction is not ensured owing to soft tissue contractures, over riding of fragments, which necessitate open reduction techniques associated with more antibiotic usage, more soft tissue dissection, reaming of the bone. The results of our study are dependent up on minimal intra operative and post operative complications following change of plan taking co morbid conditions in to consideration. The other aspect is fracture stability with change of implant.
\end{abstract}

Keywords: co morbid conditions, fracture, complications, adequate fixation.

\section{Introduction}

There are many co morbid conditions which exist before a fracture is sustained and developed or identified during the evaluation for fitness to undergo surgery. All of these conditions in one way or the other influence the time of surgery, choice of implant, antibiotics to be used and length of hospital stay.Surgical procedures are associated with a complex stress response that is proportional to the magnitude of injury, total operating time, amount of intraoperative blood loss and degree of postoperative pain. The adverse metabolic and hemodynamic effects of this stress response can present many problems in the perioperative period. Decreasing the stress response to surgery and trauma is the key factor in improving outcome and lowering the length of hospital stay as well as the total costs of patient care.It is well recognized that safe and efficient surgical and anesthesia practice requires an optimized patient. Several of the large scale epidemiological studies have indicated that inadequate preoperative preparation of the patient may be a major contributory factor to the primary causes of perioperativemortality ${ }^{(1)}$.Surgical complications occur frequently. One large study documented at least one complication in $17 \%$ of surgical patients. Surgery related morbidity and mortality generally fall into one of three categories: cardiac, respiratory and infectious complications. The overall risk for surgery related complications depends on individual factors and the type of surgical procedure. For example, advanced age places a patient at increased risk for surgical morbidity and mortality. The reason for an age related increase in surgical complications appears to correlate with an increased likelihood of underlyingdisease states in older persons.

Diseases associated with an increased risk for surgical complicationsinclude respiratory and cardiac diseases, malnutrition and diabetes mellitus. With respect to the type ofsurgery, major vascular, intraabdominal and intrathroracic surgical procedures, as well as intracranialneurosurgical procedures are frequently associated with increased perioperative morbidity andmortality. In addition, urgent and emergency procedures constitute higher risk situations thanelective, nonurgent surgery and present a limited opportunity for preoperative evaluation and treatment.

Preoperative pulmonary evaluation is important in the management of patients with lung diseasewho are undergoing elective cardiothoracic or noncardiothoracic surgery. In some instances,preoperative pulmonary evaluations may also contribute to the management of patients beingconsidered for urgent surgery. The incidence of postoperative pulmonary complications (PPCs) ishigh and is associated with substantial morbidity and mortality, and prolonged hospital stays.Perioperative pulmonary complications in patients undergoing elective noncardiothoracic surgerycan be more accurately predicted than in patients undergoing elective cardiothoracic surgery.Effective strategies to prevent complications in the postoperative period are few. Incentive spirometryand continuous positive airway pressure are the only modalities of proven benefit. Identifyingpatients who are at risk for the development of PPCs and managing their underlying modifiable risk factors aggressively prior to surgery is essential ${ }^{(2)}$.

Renal parameters influence the outcome interms of Antibiotics usage, electrolyte imbalances, Blood transfusions and length of hospital stay.Based on the analysis of reamed and undreamed procedures, early 
reamed intra medullary nail is safe in appropriately selected cases. In patients with traumatic femur fractures, early reamed intra medullary nail is associated with low hospital complications and shorter hospital stay. The rate of pulmonary complications is almost the same in the early and late group. Further prospective randomized studies with large sample size would be ideal using the information garnered from the present study ${ }^{(3)}$.

\section{Materials And Methods}

The study is designed as observational study on co morbid conditions largely influencing the pre operative preparation of the patient interms of evaluation and treatment of comorbid conditions, influence on selection of the implant, antibiotics, and length of hospital stay. The study is done with different long bone fractures. We have included the cases presented to GGH, Guntur, treated by Department of Orthopaedics from Jan 2007 to Dec 2016.The study is done with 83 patients who are diagnosed as having a co morbid condition which can influence the outcome preoperatively and post operatively. They are grouped in to three groups where in cardiac, respiratory and renal comorbid conditions whichare identified. Each co morbid conditionis evaluated and treated by the concerned medical speciality for the treatment as well as to ascertain the fitness to undergo surgery. Fourteen patients study are found to have more than one co morbid condition. Cardiac fitness is evaluated by using Echo cardiogram. The patient evaluation revealing high risk and moderate risk to undergo fracture fixation is not planned for surgery with in the first one week following trauma.

They are continued on medication till one week reevaluation is performed. The fracture fixation has been postponed till this reevaluation, no improvement in cardiac function forced to change the choice of implant and technique to save the surgery time and avoid intra operative complications.Respiratory evaluation is done to patients with pre existing COPD and Bronchial asthma. The evaluation done with Spirometry and culture sensitivity of thesputum. Patients are continued on Broncho dilators and respiratory physiotherapy till one week following trauma. The fracture fixation has been postponed till this re evaluation, no improvement in respiratory function forced to change the choice of implant and technique to save the surgery time and avoid intra operative complications. Renal evaluation is done to patients who are found to have higher values of Blood urea and Serum creatinine. Most of the patients with compromised renal function are managed conservatively and eight out of 23 cases with parenchymal disease underwent dialysis. The compromised renal status in these cases has forced the treatment plan for fracture fixation which requires minimal intervention and dissection to minimize the usage of amino glycosides. The study groups have the following case distribution: Out of 83 cases in this study group, cardiac failure is noted in 35 cases, COPD / Bronchial Asthma is seen in 21 cases, Renal failure is seen 27 cases.

Modified options for fracture fixation: the fracture patterns in this study are cases which require definitive fixation as and when the patients are fit to undergo surgery. The ideal time of fracture fixation with ideal implant is around five days, beyond which the fracture reduction is not ensured owing to soft tissue contractures, over riding of fragments, which necessitate open reduction techniques associated with more antibiotic usage, more soft tissue dissection, reaming of the bone. After the first week of re evaluation the treatment for fracture fixation is proceeded with Unreamed solid IM nails, Rush nails, Enders nails, $\mathrm{K}$ wires. The patients are mobilized with assisted ,non weight bearing and non weight lifting exercises as soon as tolerated by the patients.This option has been chosen, because these fixations require lesser soft tissue dissection except at entry points, no reaming, no dependence on more antibiotics.

\section{Results}

The results of our study are dependent up on minimal intra operative and post operative complications following change of plan taking co morbid conditions in to consideration. The other aspect is fracture stability with change of implant.Going by the co morbid conditions included in the study, Cardiac failure has more peri operative complications compared to Renal failure and COPD/Bronchial asthma. Out of 35 cases with cardiac failure in the study 23 of them developed hypotension, in the immediate post operative period, requiring them to be shifted for ICU management. They are in turn managed with inotropics and Blood transfusions with packed cells.

Out of 27 renal failure cases 21 of them showed further improvement and 6 of them again required dialysis. All the six cases developed post operative infections with superficial infections. Out of 21 COPD/Bronhial asthma patients, all of them are given nebulisation till seven days following surgery, who gradually showedimprovement. None of the cases in cardiac failure group and COPD/Bromchial asthma had any post operative infections.Results depending on fracture fixation differ from one fracture pattern to the other. Fractures treated with enders nailing showed better fracture approximation than other fixation devices in moderate comminution. The fractures fixed with Rush nails showed rotational deformity which is beyond the inherent stability offered the implant design. The solid IM nailing is observed to be providing better approximation, where in, even the rotational deformity could be controlled.All the cases with co morbid conditions demand care for minimizing deformity which require a bigger time consuming procedure. 


\section{Figures and Tables}

Fig.1Peri operative cardiac complications

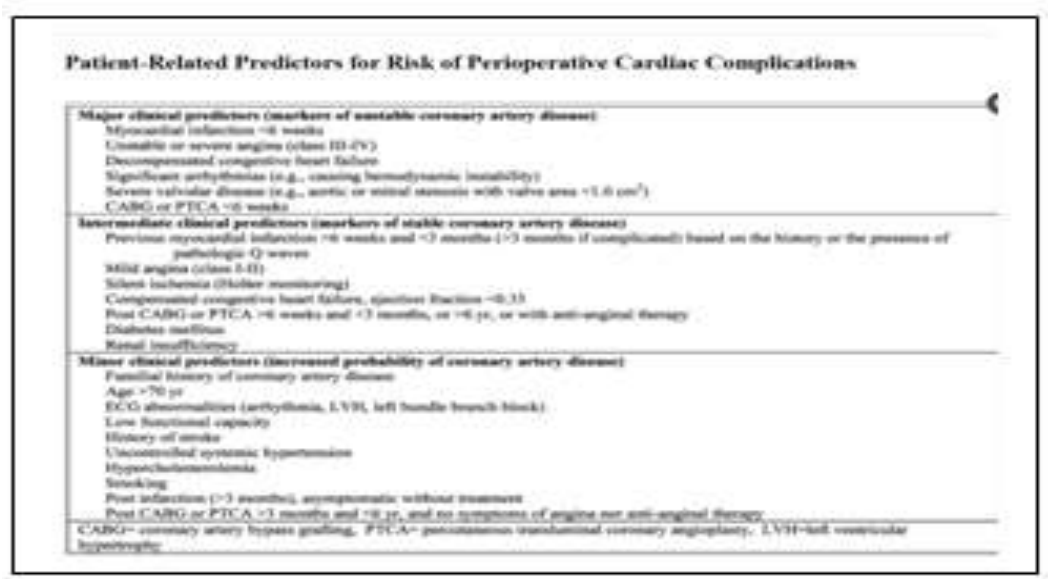

Fig.2 : Predictors of cardiac complications

\begin{tabular}{|c|c|}
\hline \multicolumn{2}{|r|}{ Surgery-Related Predictors for Risk of Perioperative Cardiac Complic: } \\
\hline & $\begin{array}{l}\text { High risk procedures (eardiae complication rate }>5 \% \text { ) } \\
\text { Emergency surgery } \\
\text { Aortic and major vascular surgery } \\
\text { Prolonged surgical procedures with large fluid shifts or blood loss } \\
\text { Unstable hemodynamic situations }\end{array}$ \\
\hline & $\begin{array}{l}\text { Intermediate risk procedures (cardiac complication rate 1-5\%) } \\
\text { Abdominal or thoracic surgery } \\
\text { Neurosungery } \\
\text { ENT procedures } \\
\text { Minor vascular surgery, including carotid endarterectomy } \\
\text { Orthopedic surgery } \\
\text { Prostatectomy }\end{array}$ \\
\hline i & $\begin{array}{l}\text { Low risk procedures (cardiac complication rate }<1 \% \text { ) } \\
\text { Breast surgery } \\
\text { Superficial surgery } \\
\text { Eye surgery } \\
\text { Endoscopic procedures } \\
\text { Plastic and reconstructive surgery } \\
\text { Ambulatory surgery }\end{array}$ \\
\hline
\end{tabular}

FIG.1 Inadequate fixation

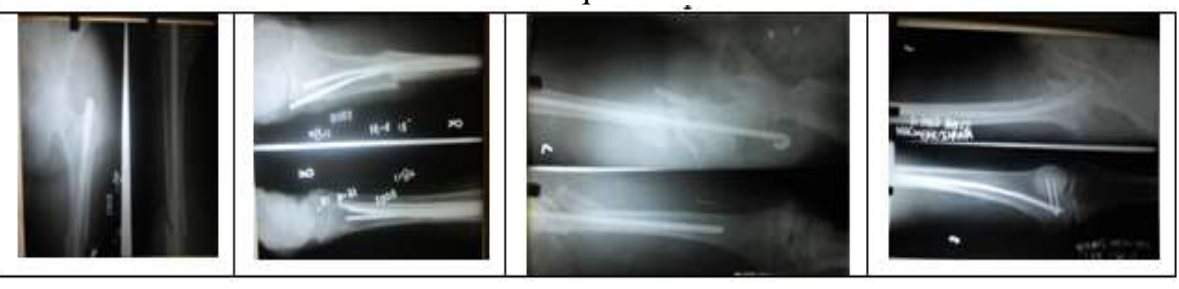

Fig.2: Adequate fixation

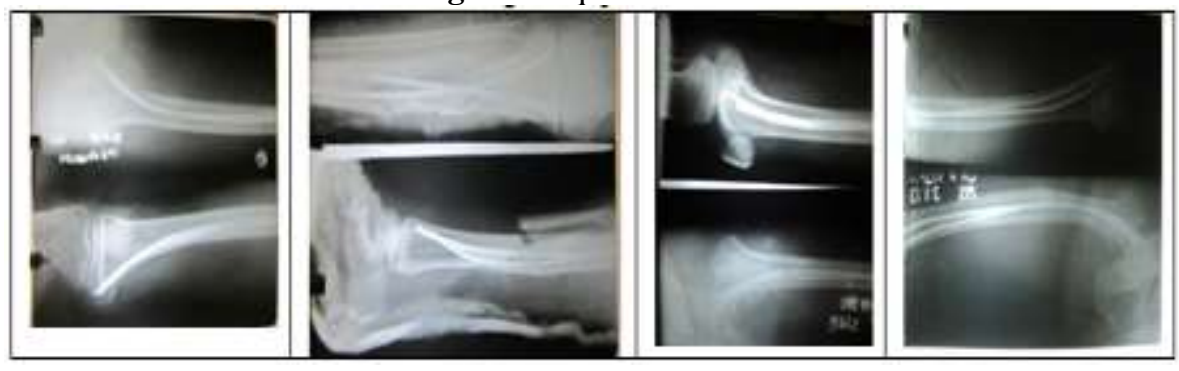


Table 1: Case and Co morbid condition distribution

\begin{tabular}{|l|c|c|c|c|}
\hline Fracture & \multicolumn{5}{|c|}{ Co Morbid Condition } \\
\hline & & Cardiac Failure & COPD/ Bronchial Asthma & Renal Failure \\
\hline Femur & 29 & 21 & 3 & 5 \\
\hline Tibia & 35 & 8 & 15 & 12 \\
\hline Humerus & 19 & 6 & 3 & 10 \\
\hline
\end{tabular}

Table 2: Choice of implant as per co morbid condition instead of Reamed IMN

\begin{tabular}{|l|c|c|c|}
\hline Co Morbid Condition & Femur & Tibia & Humerus \\
\hline Cardiac Failure & Enders Nail & Solid Im & Rusch/ Enders Nail \\
\hline COPD/ Bronchial Asthama & Enders Nail & Solid IM/Enders & Rusch/ Enders Nail \\
\hline Renal Failure & Enders & Solid IM/Enders & Rusch/ Enders Nail \\
\hline
\end{tabular}

\section{Conclusion}

In our study it is observed that co morbid conditions will force the surgeon to opt for choices with least per and post operative complications in fixation of fractures. The evaluation of the patient from the beginning should be able to rule out co morbid conditions. These conditions will delay fracture treatment with definitive fixation, not only that the available other options may not give a proper reduction.Out of all the choices, Solid IM nailing will give better fixation in compromised planning taking into consideration of co morbid conditions. The next better option is enders nail which can fill the medullary canalcontrolling rotational deformity in the initial days till the implant loosening occur. Rush nails can maintain the length of the limb but still the fracture stability is questionable and rotational deformity also.

The co morbid conditions, the moment they are diagnosed, should be attended to along with the care of the fracture to avoid further displacement of the fracture which may not be corrected by opting for available choices. Proper limb elevation, and splinting are required taking with tractions to avoid further displacement, even if the patient gets improvement ideal fixation will not be possible with minimal dissection.

\section{References}

Journal Papers:

[1]. Hippokratia. 2007 JanMar; 11(1): 13-21. PMCID: PMC2464262 Preoperative evaluation and preparation for anesthesia and surgery

[2]. A Zambouri 4th Dept of Anesthesiology, "Hippokratio" G. H., Thessaloniki, Greece Corresponding author: Zambouri A, 4th Dept of Anesthesiology, "Hippokratio" G. H., Thessaloniki, Greece, tel: 2310346131 athzab @ yahoo.gr

[3]. Mt Sinai J Med. 2005 May;72(3):18592. Preoperative cardiovascular evaluation for noncardiac surgery. Maddox TM .

[4]. Recent Advances in Chest Medicine | November 2007 Preoperative Evaluation of the Patient With Pulmonary Disease Srinivas R. Bapoje, MD, MPH; Julia Feliz Whitaker, MD; Tara Schulz, MD; Eugene S. Chu, MD; Richard, Chest. 2007;132(5):16371645. doi:10.1378/chest.070347

[5]. Orthopade. 1996 Jun;25(3):2929. [Systemic complications in intramedullary nailing]. WendaK, Runkel M.

[6]. Int J CritIllnInj Sci. 2016 JulSep;6( 3):143147. Early and late intramedullary nailing of femur fracture: A single

[7]. center experience. Alobaidi AS, AlHassani A, ElMenyar A, Abdelrahman H, Tuma M, AlThani H, Aldosari MA .ci Rep. 2016 Jul 26;6:30566. doi: 10.1038/srep30566. Early intramedullary nailing of femoral shaft fracture on outcomes in patients with severe chest injury: A metaanalysis. Jiang M, Li C, Yi C, Tang S .

[8]. Am J Orthop (Belle Mead NJ). 2016 Nov/Dec;45(7):E515E521. Fat Embolism Syndrome With Cerebral Fat Embolism Associated With LongBone Fracture. DeFrodaSF ,Klinge SA.

[9]. J Am AcadOrthop Surg. 2000 MarApr;8( 2):8390. Intramedullary nailing of the femur: reamed versus nonreamed.BrumbackRJ ,Virkus WW.

[10]. ClinOrthopRelat Res. 1998 Feb;(347):5761.Treatment of femoral fractures in the multiply injured patient withthoracic injury.Bone LB , Anders MJ, Rohrbacher BJ. 